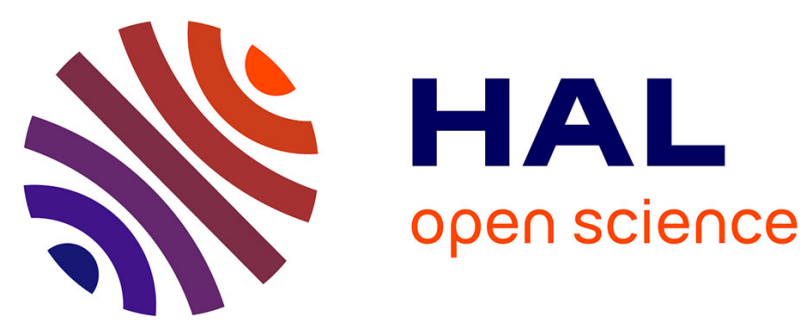

\title{
Correlation between cardio-pulmonary exercise test variables and ă health-related quality of life among children with congenital heart $\breve{a}$ diseases
}

P. Amedro, M. C. Picot, S. Moniotte, R. Dorka, H. Bertet, S. Guillaumont, C. Barrea, M. Vincenti, G. de La Villeon, C. Bredy, et al.

\section{To cite this version:}

P. Amedro, M. C. Picot, S. Moniotte, R. Dorka, H. Bertet, et al.. Correlation between cardio-pulmonary exercise test variables and $\breve{a}$ health-related quality of life among children with congenital heart $\breve{a}$ diseases. International Journal of Cardiology, 2016, 203, pp.1052-1060. 10.1016/j.ijcard.2015.11.028 . hal-02383830

\section{HAL Id: hal-02383830 \\ https://hal.science/hal-02383830}

Submitted on 28 Nov 2019

HAL is a multi-disciplinary open access archive for the deposit and dissemination of scientific research documents, whether they are published or not. The documents may come from teaching and research institutions in France or abroad, or from public or private research centers.
L'archive ouverte pluridisciplinaire HAL, est destinée au dépôt et à la diffusion de documents scientifiques de niveau recherche, publiés ou non, émanant des établissements d'enseignement et de recherche français ou étrangers, des laboratoires publics ou privés. 


\title{
Correlation between cardio-pulmonary exercise test variables and health-related quality of life among children with congenital heart diseases 2 ?
}

P. Amedro a,d,e, $\square$, M.C. Picot b,f, S.Moniotte c, R. Dorka a, H. Bertet c,f, S.Guillaumont a,g, C. Barrea c, M. Vincenti a,d, G. De La Villeon a,g, C. Bredya, C. Soulatges a, M. Voisin a, S.Matecki d,h, P. Auquier e

a Pediatric and Congenital Cardiology Department, University Hospital, Montpellier, France b Epidemiology and Clinical Research Department, University Hospital, Montpellier, France c Pediatric and Congenital Cardiology Department, St-Luc University Hospital, UCL, Brussels, Belgium

d Physiology and Experimental Biology of Heart and Muscles Laboratory - PHYMEDEXP, UMR CNRS 9214 - INSERM U1046, University of Montpellier, Montpellier, France

e Self-perceived Health Assessment Research Unit, EA3279, Public Health Department, Mediterranean Medical School, Marseille, France

f Clinical Investigation Center, INSERM-CIC 1411, Montpellier, France

g Pediatric Cardiology and Rehabilitation Unit, St-Pierre Institute, Palavas-Les-Flots, France

h Pediatric Functional Exploration Laboratory, Physiology Department, University Hospital,

Montpellier, France

The authors take responsibility for all aspects of the reliability and freedom from bias

of the data presented and their discussed interpretation.

$\square$ Corresponding author at: Pediatric and Congenital Cardiology Department,

Montpellier University Hospital, 371 Avenue du Doyen Giraud, 34295Montpellier, France.

E-mail address: p-amedro@chu-montpellier.fr (P. Amedro).

\begin{abstract}
Absctract
Background: Health-related quality of life (HR-QoL) stands as a determinant "patient-related outcome" and correlateswith cardio-pulmonary exercise test (CPET) in adults with chronic heart failure orwith a congenital heart disease (CHD). No such correlation has been established in pediatric cardiology.

Methods and results: 202 CHD children aged 8 to 18 performed a CPET (treadmill $\mathrm{n}=96$, cycle-ergometer $\mathrm{n}=106)$. CHD severity was stratified into 4 groups. All children and parents filled out the Kidscreen HR-QoL questionnaire. Peak VO2, anaerobic threshold (AT), and oxygen pulse followed a downward significant trendwith increasing CHD severity and conversely for VE/VCO2 slope. Self-reported and parent-reported physical well-being HRQoL scores correlated with peak VO2 (respectively $\mathrm{r}=0.27, \mathrm{p}$ b 0.0001 and $\mathrm{r}=0.43, \mathrm{p} \mathrm{b}$ $0.0001)$, percentage of predicted peak VO2 $(\mathrm{r}=0.28, \mathrm{p}=0.0001$ and $\mathrm{r}=0.41, \mathrm{p} \mathrm{b} 0.0001)$, and percentage of predicted VO2 at AT $(r=0.22$, p b 0.01 and $r=0.31, p$ b 0.0001). Significant correlations were also observed between several HRQoL dimensions and dead space to tidal volume ratio (VD/VT), oxygen uptake efficiency slope (OUES), oxygen pulse but never with VE/VCO2 slope. The strongest correlations were observed in the treadmill group, especially between peak VO2 and physical well-being for parents $(\mathrm{r}=0.57, \mathrm{p} \mathrm{b} 0.0001)$ and self $(\mathrm{r}=0.40$, p b 0.0001) reported HR-QoL.
\end{abstract}

Conclusions: Peak VO2 and AT are the two CPET variables that best correlated with HRQoL in this large pediatric cohort, parents' reports being more accurate. If HR-QoL is 
involved as a "PRO" in a pediatric cardiology clinical trial, we suggest using parents related physical well-being HR-QoL scores.

\section{Introduction}

Quality of life (QoL) now stands as a determinant "patient-related outcome" [1] and is frequently used as a secondary endpoint in cardiovascular clinical trials [2]. The "overall life satisfaction" [3] being a quite subjective definition, most studies refer to "health-related quality of life" (HR-QoL) and use multidimensional questionnaires including at least physical, social, psychological, mental, and functional aspects [4]. Validation of these HR QoL instruments is based on good psychometric properties such as validity, reproducibility, and sensitivity to change $[3,5,6]$. Good correlations between HR-QoL and the severity of the disease are a prerequisite for these clinical studies. TheWorld Health Organization stated that functional capacity explorations best reflect the impact of chronic diseases on QoL [7]. In cardiology, cardio-pulmonary exercise test (CPET) is considered as a "gold standard" to quantify the disease severity. Indeed, in chronic heart failure, CPET (peak VO2 and VE/VCO2 slope) correlates with both HR-QoL and prognosis [8-11]. Similar results have been found in adults with CHD [12-16]. However, no data about correlations between HRQoL and physical capacity from a large CPET cohort is available in pediatric cardiology. This study therefore aimed to identify the correlations between self and parents reported HR-QoL and CPET variables on a cohort of children with CHD, considering the severity of the disease.

\section{Method}

\subsection{Study design}

This prospective cross-sectional studywas carried out between April 2009 and October 2011 (18 months) in two tertiary care pediatric and congenital cardiology departments (Montpellier University Hospital, France and Brussels Saint-Luc University Hospital, Belgium).Weincluded all children who performed a complete CPET among our previously reported QoL study [17].

\subsection{Patients' population}

Children aged 8 to 18 years with CHD were prospectively recruited during a regular pediatric cardiology outpatient visit. Children without CHD (cardiac exploration for syncope/faint, thoracic pain, palpitations, dyspnea, etc.) were not eligible. We did not include children with any other severe chronic disease (neuro-developmental disorder, chronic renal or respiratory failures) and children and/or families unable to understand the QoL questionnaire. Children with recent surgical or catheter cardiac intervention (6 months delay) and hospitalized children were temporary excluded but could be recruited during their following annual outpatient visit.

\subsection{QoL questionnaires}

Right before CPETwas performed, children filled in the Kidscreen-52 questionnaire, a generic validated pediatric HR-QoL instrument $[18,19]$. The Kidscreen is a reliable, valid, sensitive, and conceptually/linguistically appropriate HR-QoL measure in 38 countries/languages [20]. Designed for healthy and chronically ill children and adolescents aged between 8 and 18 years, it measures 10 dimensions: physical wellbeing (5 items), psychological well-being (6 
items), moods \& emotions (7 items), self-perception (5 items), autonomy (5 items), parent relations \& home life (6 items), social support \& peers (6 items), school environment (6 items), social acceptance (bullying) (3 items), financial resources (3 items). At the same time, in a separate room, before they learned the results of their child's CPET, parents filled in the Kidscreen-27 proxy questionnaire, which measures 5 dimensions: physical well-being (5 items), psychological well-being (7 items), autonomy \& parent relations ( 7 items), social support \& peers (4 items), and school environment(4 items) [21,22]. HR-QoL scores are given for each dimension and vary from 0 (lowest HR-QoL) to 100 (highest HR-QoL).

Table 1 CHD severity classification [32].

Severity class 1

Severity class 2

Severity class 3

Severity class 4
Mild CHD requiring no therapy or effectively treated non-operatively (catheter therapy)

Moderate CHD requiring no therapy or surgically corrected (curative)

Surgically treated CHD with significant residua or need for additional surgery

Complex or severe CHD, uncorrectable or palliated (includes single ventricle)

Table 2 Socio-demographic characteristics.

\begin{tabular}{|c|c|c|c|c|}
\hline & \multirow{2}{*}{$\begin{array}{c}\text { CHD total } \\
\mathrm{N}=202\end{array}$} & \multicolumn{2}{|l|}{ CHD } & \multirow[t]{2}{*}{ p-value } \\
\hline & & $\begin{array}{r}\text { France } \\
\mathrm{N}=106\end{array}$ & $\begin{array}{l}\text { Belgium } \\
\mathrm{N}=96\end{array}$ & \\
\hline Sex ratio (male/female) & 1.9 & 2.2 & 1.7 & 0.34 \\
\hline Age (mean (SD)) & $12.4(3.0)$ & $12.2(2.9)$ & $12.6(3.2)$ & 0.39 \\
\hline School class & & & & 0.38 \\
\hline Elementary school & $62(31 \%)$ & $34(33 \%)$ & $28(29 \%)$ & \\
\hline Middle school & $95(48 \%)$ & $51(49 \%)$ & $44(46 \%)$ & \\
\hline High school & $26(13 \%)$ & $13(13 \%)$ & $13(13 \%)$ & \\
\hline Specialized education & $16(8 \%)$ & $5(5 \%)$ & $11(12 \%)$ & \\
\hline School level & & & & 0.41 \\
\hline Normal & $131(66 \%)$ & $68(65 \%)$ & $63(66 \%)$ & \\
\hline Advanced & $2(1 \%)$ & $2(2 \%)$ & $0(0 \%)$ & \\
\hline Repeated & $64(32 \%)$ & $32(31 \%)$ & $32(34 \%)$ & \\
\hline Dropout & $2(1 \%)$ & $2(2 \%)$ & $0(0 \%)$ & \\
\hline
\end{tabular}

Table 3 Medical characteristics.

CHD

Severity Class

\begin{tabular}{clr}
\hline Total & France & Belgium \\
$\mathrm{N}=202$ & $\mathrm{~N}=106$ & $\mathrm{~N}=96$
\end{tabular}


Class 1

Class 2

Class 3

Class 4

Total

CHD

Heterotaxy

Anomalies of venous return

Anomalies of the atria and interatrial

Communications

Anomalies of the atrioventricular junctions and valves

Complex anomalies of the atrioventricular

Connections

Functionally univentricular hearts

Ventricular septal defects (VSD)

Anomalies of the ventricular outflow tracts

Anomalies of the extrapericardial arterial

Trunks

Congenital anomalies of the coronary

Arteries

Other CHD (HCM, DCM, LQTS, CPVT)a

Invasive treatment

Cardiac surgery

Patients with at least one cardiac surgery

1 intervention

2 interventions

$\geq 3$ interventions

Total

Intervention catheter

Patients with at least one intervention

1 intervention

2 interventions

$\geq 3$ interventions

Total

Medical treatment

Patients with at least one treatment

Beta-blockers

Angiotensin-converting enzyme inhibitors

Calcium channel blockers

Diuretics

Oral anticoagulants

\begin{tabular}{|c|c|c|}
\hline $45(23 \%)$ & $22(21 \%)$ & $23(24 \%)$ \\
\hline $28(14 \%)$ & $13(12 \%)$ & $15(15 \%)$ \\
\hline $99(49 \%)$ & $59(57 \%)$ & $40(42 \%)$ \\
\hline $28(14 \%)$ & $10(10 \%)$ & $18(19 \%)$ \\
\hline $200(100 \%)$ & $104(100 \%)$ & $96(100 \%)$ \\
\hline $0(0 \%)$ & $0(0 \%)$ & $0(0 \%)$ \\
\hline $4(1 \%)$ & $2(1 \%)$ & $2(1 \%)$ \\
\hline $14(5 \%)$ & $5(3.5 \%)$ & $9(5 \%)$ \\
\hline $16(5 \%)$ & $4(3 \%)$ & $12(7 \%)$ \\
\hline $10(3 \%)$ & $1(1 \%)$ & $9(5 \%)$ \\
\hline $53(17 \%)$ & $17(12 \%)$ & $36(22 \%)$ \\
\hline $17(6 \%)$ & $11(8 \%)$ & $6(4 \%)$ \\
\hline $140(46 \%)$ & $64(46 \%)$ & $76(46 \%)$ \\
\hline $38(12 \%)$ & $26(19 \%)$ & $12(7 \%)$ \\
\hline $6(2 \%)$ & $4(3 \%)$ & $2(1 \%)$ \\
\hline $8(3 \%)$ & $5(3.5 \%)$ & $3(2 \%)$ \\
\hline $144(72 \%)$ & $77(73 \%)$ & $67(70 \%)$ \\
\hline $76(53 \%)$ & $50(65 \%)$ & $26(39 \%)$ \\
\hline $30(21 \%)$ & $19(25 \%)$ & $11(16 \%)$ \\
\hline $38(26 \%)$ & $8(10 \%)$ & $30(45 \%)$ \\
\hline $144(100 \%)$ & $77(100 \%)$ & $67(100 \%)$ \\
\hline $64(32 \%)$ & $20(19 \%)$ & $44(47 \%)$ \\
\hline $43(67 \%)$ & $17(85 \%)$ & $26(59 \%)$ \\
\hline $8(13 \%)$ & $2(10 \%)$ & $6(14 \%)$ \\
\hline $13(20 \%)$ & $1(5 \%)$ & $12(27 \%)$ \\
\hline $64(100 \%)$ & $20(100 \%)$ & $44(100 \%)$ \\
\hline $144(72 \%)$ & $77(73 \%)$ & $67(70 \%)$ \\
\hline $60(30 \%)$ & $29(28 \%)$ & $31(32 \%)$ \\
\hline $11(18 \%)$ & $7(24 \%)$ & $4(13 \%)$ \\
\hline $6(10 \%)$ & $1(3 \%)$ & $5(16 \%)$ \\
\hline $2(3 \%)$ & $2(7 \%)$ & $0(0 \%)$ \\
\hline $5(8 \%)$ & $3(10 \%)$ & $2(6 \%)$ \\
\hline $14(23 \%)$ & $6(21 \%)$ & $8(26 \%)$ \\
\hline
\end{tabular}

Medical device

Pacemaker

$5(2 \%)$

$1(1 \%)$

$1(1 \%)$ $\begin{array}{ll}1(1 \%) & 4(4 \%) \\ 1(1 \%) & 0(0 \%) \\ 0(0 \%) & 1(1 \%)\end{array}$ 
a HCM: hypertrophic cardiomyopathy. DCM: dilated cardiomyopathy. LQTS: long QT syndrome. CPVT: catecholaminergic polymorphic ventricular tachycardia.

\subsection{CPET procedures}

Both university hospitals are referral tertiary care pediatric and congenital cardiology centers with regular practice of CPET in the follow-up of children and adults with CHD. Children referred to one of the two CPET laboratories in their routine follow-up were offered to participate to the study. The investigator coordinator of the study participated to CPET in both centers where procedures were harmonized before the study started. Both laboratories used the same following devices: pediatric face masks (Hans Rudolph), calibrated gas analyzer (Oxycon Pro, Jaeger), breath-to-breath measurements software (Windows 98, Jaeger), 12-lead ECG equipment (Cardiosoft, GE Healthcare), pulse oximeter (Nellcor), manual sphygmomanometer with adapted pediatric cuffs.

Systematic spirometry using common gas device (Oxycon Pro, Jaeger) was performed before exercise test with flow volume curve and measurement of forced expiratory volume in $1 \mathrm{~s}$ (FEV1), forced vital capacity (FVC), FEV1/FVC ratio (FEV1\%), with normalization to theoretical values [23].

Both centers used CPET pediatric protocols adapted to CHD children [24]. French center used a cycle-ergometer protocol: 1-min rest, 3-min warm-up (10 to 20W), increments of 10, 15 or 20Weach minute, pedaling rate of 60 to $80 \mathrm{rpm}, 3$-min active recovery (20 watts), 2-min rest. Belgian center used a treadmill modified Bruce protocol: 1 min rest, 3-min warm-up $(1 \mathrm{~km} / \mathrm{h}$, slope $0 \%$ ), increments every $2 \mathrm{~min}$ in terms of speed (from 2.5 to $10.5 \mathrm{~km} / \mathrm{h}$ ) and slope (from 3 to $18 \%), 3$-min active recovery $(2.5 \mathrm{~km} / \mathrm{h}, 3 \%$ slope $), 2$-min rest.

Exercise was pursued until the limit of the child's tolerance was reached, despite verbal encouragement.

The following CPET variables were measured: oxygen uptake (VO2; $\mathrm{ml} / \mathrm{kg} / \mathrm{min}$ ), carbon dioxide production $(\mathrm{VCO} 2 ; \mathrm{ml} / \mathrm{kg} / \mathrm{min})$, respiratory exchange ratio $(\mathrm{RER}=\mathrm{VCO} 2 / \mathrm{VO} 2)$, minute ventilation (VE; breaths/min), ventilatory equivalent for oxygen (VE/VO2), ventilator equivalent for carbon dioxide (VE/VCO2), dead space to tidal volume ratio (VD/VT), heart rate (HR; beats per minute — bpm), maximum load (watts), and oxygen pulse (VO2/HR; ml). For all CPET performed within the study, the same investigator coordinator manually calculated the peak oxygen uptake (peak VO2), the ventilatory anaerobic threshold (AT) using Beaver's method [25], the ventilation efficiency (VE/VCO2 slope with VE $=$ slope $\times$ $\mathrm{VCO} 2+b)$ and the oxygen uptake efficiency slope (OUES with VO2 $=$ OUES $\times \log 10 \mathrm{VE}+$ b) [26-28]. Peak VO2 and AT were normalized in percentage of predicted peak VO2 using normal values from Wasserman and Cooper [29-31].

\subsection{CHD severity}

In adult population, heart failure is classically stratified upon the NYHA functional class but this classification does not apply to pediatric cardiology and is rarely used by physicians. We chose to classify our pediatric CHD population into 4 severity groups using previous classification from Uzark et al. (Table 1) [32]. ACC-CHDclassification fromHouyel et al. was used to define the type of malformation [33]. 


\subsection{Formal aspects}

The study protocol conformed to the ethical guidelines of the 1975 Declaration of Helsinki. It was approved by Ethics Committees in France (South Mediterranean IV) and Belgium (UCL Medical School) and was registered on ClinicalTrials.gov (number NCT01202916). Informed consent was obtained from all parents.

\subsection{Statistics}

The study population was described withmeans and standard deviations (SD) for quantitative variables and with frequencies for qualitative variables. The continuous variable distributions were tested with the Shapiro-Wilk statistic. Quantitative variables were compared with Student's t-test when the distribution was Gaussian, and with the Mann-Whitney test otherwise. For qualitative variables, groups were compared with Chi-square or Fisher test. The two-sided Jonckheere trend test investigated the existence of a trend according to the severity class. ANOVA or Kruskal-Wallis tests were used for comparisons between severity classes. To measure the strength and direction of the relationships between HR-QoL scores and CPET variables, a Pearson or Spearman correlation coefficient was used. And to test the linearity of the relation between the main CPET variables and HR-QoL scores, the normality of residues was tested with Shapiro-Wilk test. Sensitivity analysis for age and gender was performed. Statistical significance was set at 0.05 and analyses were performed using SAS version 9 (SAS Institute, Cary, NC).

Table 4 CPET results: stratification on CHD severity.

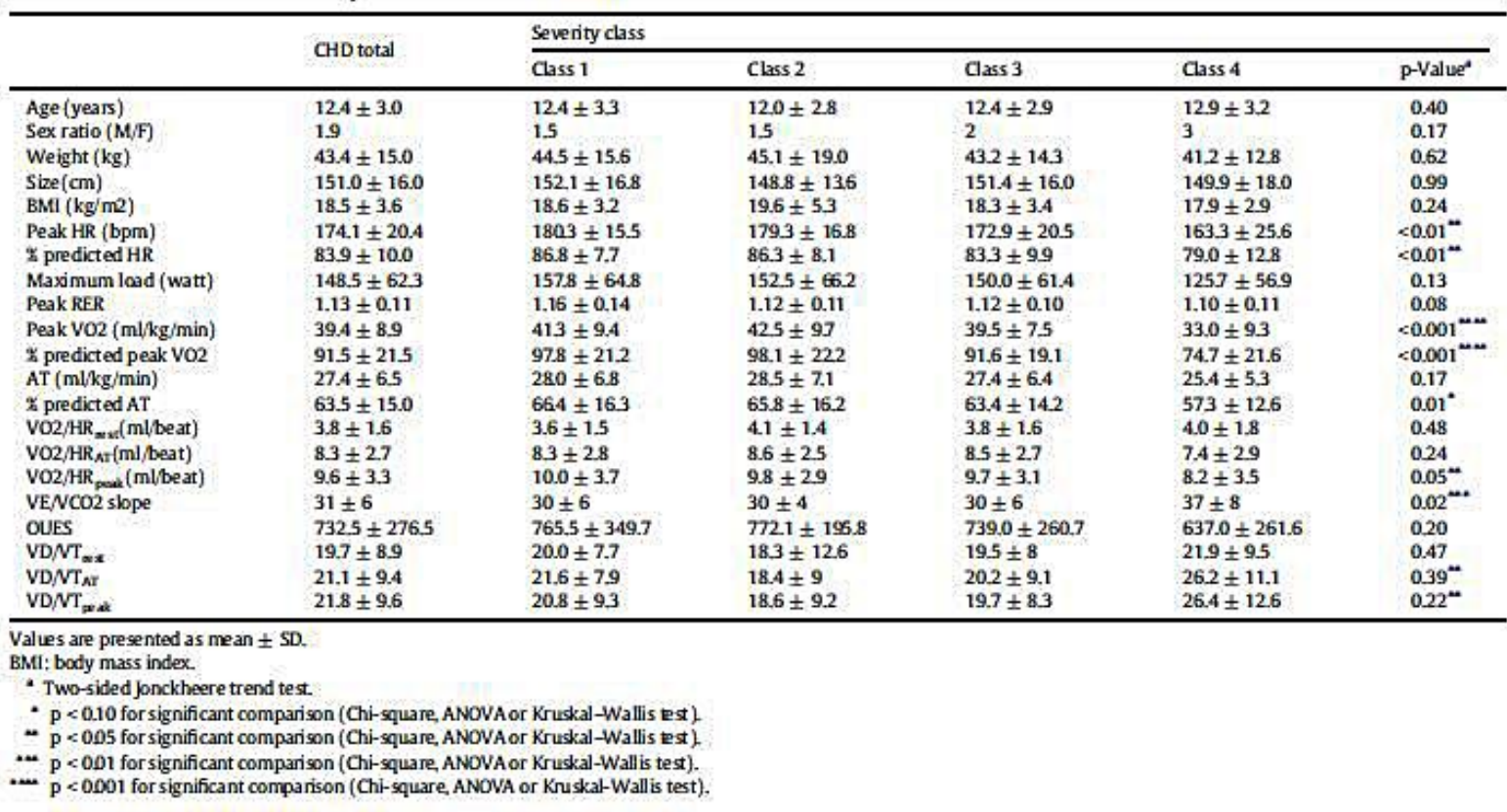

\section{Results}

\subsection{Population}

During the study period, 261 CHD children aged 8 to 18 years were referred for CPET; 25 were not eligible because of recent intervention catheter procedure $(n=5)$, recent surgical 
procedure $(n=7)$, impossibility to answer the questionnaire (Down syndrome $n=5$, DiGeorge syndrome $n=5)$, other significant disease $(n=3) ; 35$ families refused to participate.

Table 5 CPET results: stratification on gender and type of protocol.

\begin{tabular}{|c|c|c|c|c|c|c|c|}
\hline & \multirow{2}{*}{$\frac{\text { CHD }}{\text { total } N=202}$} & \multicolumn{3}{|l|}{ GENDER } & \multicolumn{3}{|l|}{ CPET protocol } \\
\hline & & Fernale $N=69$ & Male $N=133$ & $\mathrm{p}$-Value & Cycle ergometer $N=106$ & Treadmill $N=96$ & p-Value \\
\hline Age (years) & $12.4 \pm 3.0$ & $12.8 \pm 3.1$ & $122 \pm 3.0$ & 0.14 & $12.2 \pm 2.9$ & $126 \pm 3.2$ & 0.39 \\
\hline Sex ratio $(\mathrm{M} / \mathrm{F})$ & 1.9 & $-\quad-2+$ & $-2-2$ & - & 22 & 1.7 & 0.34 \\
\hline Weight $(\mathrm{kg})$ & $43.4 \pm 15.0$ & $4.8 \pm 13.5$ & $43.2 \pm 15.7$ & 0.41 & $43.3 \pm 15.0$ & $438 \pm 15.1$ & 0.65 \\
\hline Size $(\mathrm{cm})$ & $151.0 \pm 16.0$ & $150 \pm 15$ & $151 \pm 17$ & 0.85 & $151 \pm 16$ & $152 \pm 16$ & 0.54 \\
\hline $\operatorname{BMI}\left(\mathrm{kg} / \mathrm{m}^{2}\right)$ & $18.5 \pm 3.6$ & $18.9 \pm 3.4$ & $183 \pm 3.7$ & 0.11 & $18.5 \pm 3.7$ & $18.5 \pm 3.5$ & 0.96 \\
\hline Peak HR (bpm) & $174.1 \pm 20.4$ & $176.4 \pm 19.2$ & $172.8 \pm 21.0$ & 0.23 & $1710 \pm 203$ & $177.8 \pm 20.0$ & 0.001 \\
\hline x predicted HR & $83.9 \pm 10.0$ & $85.2 \pm 9.5$ & $831 \pm 10.2$ & 0.15 & $82.3 \pm 9.8$ & $85.8 \pm 9.9$ & $<0.001$ \\
\hline Maximum bad (watt) & $148.5 \pm 62.3$ & $139.9 \pm 60.0$ & $1529 \pm 63.3$ & 0.13 & $1323 \pm 43.7$ & $164.1 \pm 73.0$ & 0.01 \\
\hline Peak RER & $1.13 \pm 0.11$ & $1.14 \pm 0.10$ & $1.13 \pm 0.12$ & 0.34 & $1.12 \pm 0.12$ & $1.14 \pm 0.10$ & 0.06 \\
\hline Peak VO2 (ml/kg/min) & $39.4 \pm 8.9$ & $34.5 \pm 7.5$ & $41.9 \pm 8.5$ & $<0.001$ & $39.3 \pm 8.3$ & $39.5 \pm 9.5$ & 0.89 \\
\hline \& predicted peak VO2 & $91.5 \pm 21.5$ & $90.5 \pm 20.8$ & $92,0 \pm 219$ & 1.00 & $924 \pm 203$ & $90.5 \pm 22.8$ & 0.54 \\
\hline $\begin{array}{l}\mathrm{AT}(\mathrm{ml} / \mathrm{kg} / \mathrm{min}) \\
\aleph_{\mathrm{s}} \text { predicted AT }\end{array}$ & $\begin{array}{l}27.4 \pm 6.5 \\
63.5 \pm 15.0\end{array}$ & $\begin{array}{l}24.0 \pm 5.3 \\
63.4 \pm 14.5\end{array}$ & $\begin{array}{l}20.0 \pm 6.4 \\
6.6 \pm 15.2\end{array}$ & $\begin{array}{l}<0.001 \\
0.92\end{array}$ & $\begin{array}{l}26.9 \pm 6.2 \\
63.4 \pm 14.8\end{array}$ & $\begin{array}{l}27.9 \pm 6.7 \\
637 \pm 15.2\end{array}$ & $\begin{array}{l}0.31 \\
0.87\end{array}$ \\
\hline $\mathrm{VO} 2 / \mathrm{HR}_{\mathrm{sx}}(\mathrm{ml} /$ beat $)$ & $3.8 \pm 1.6$ & $36 \pm 1.3$ & $40 \pm 1,7$ & 0.10 & $3.8 \pm 1.7$ & $3.9 \pm 1.4$ & 0.38 \\
\hline $\mathrm{VO}_{2} / \mathrm{HR}_{\text {ain }}$ (ml/beat) & $8.3 \pm 27$ & $73 \pm 20$ & $88 \pm 2.9$ & 0.001 & $8.3 \pm 2.8$ & $8.3 \pm 2.7$ & 0.91 \\
\hline $\mathrm{V} 02 / \mathrm{HR}_{\text {pouk }}(\mathrm{m} / \mathrm{beat})$ & $9.6 \pm 3.3$ & $8.3 \pm 2.3$ & $102 \pm 3.5$ & $<0.001$ & $9.7 \pm 3.3$ & $9.4 \pm 3.3$ & 0.60 \\
\hline VE/NCO2 slope & $31 \pm 6$ & $32 \pm 8$ & $31 \pm 6$ & 0.84 & - & - & - \\
\hline OUES & $732.5 \pm 276.5$ & $683.1 \pm 2415$ & $762.5 \pm 2937$ & 0.19 & - & - & - \\
\hline $\mathrm{VD} / \mathrm{VT}_{\text {tion }}$ & $19.7 \pm 8.9$ & $20.7 \pm 10.2$ & $191 \pm 8.2$ & 0.43 & $18.0 \pm 9.7$ & $212 \pm 79$ & $<0.01$ \\
\hline $\mathrm{VD} / \mathrm{NT}_{\mathrm{Ar}}$ & $21,1 \pm 9.4$ & $22.2 \pm 9.0$ & $204 \pm 9.5$ & 0.19 & $16.9 \pm 7.1$ & $24.5 \pm 9.6$ & $<0.001$ \\
\hline $\mathrm{VD} / \mathrm{NT}_{\mathrm{ptak}}$ & $21.8 \pm 9.6$ & $21.9 \pm 10.7$ & $201 \pm 8.9$ & 039 & $16.8 \pm 5.2$ & $240 \pm 11.1$ & $<0.001$ \\
\hline
\end{tabular}

Values are presented as mean \pm SD. Significant $p$-values $<0,05$ are marked in bolk.

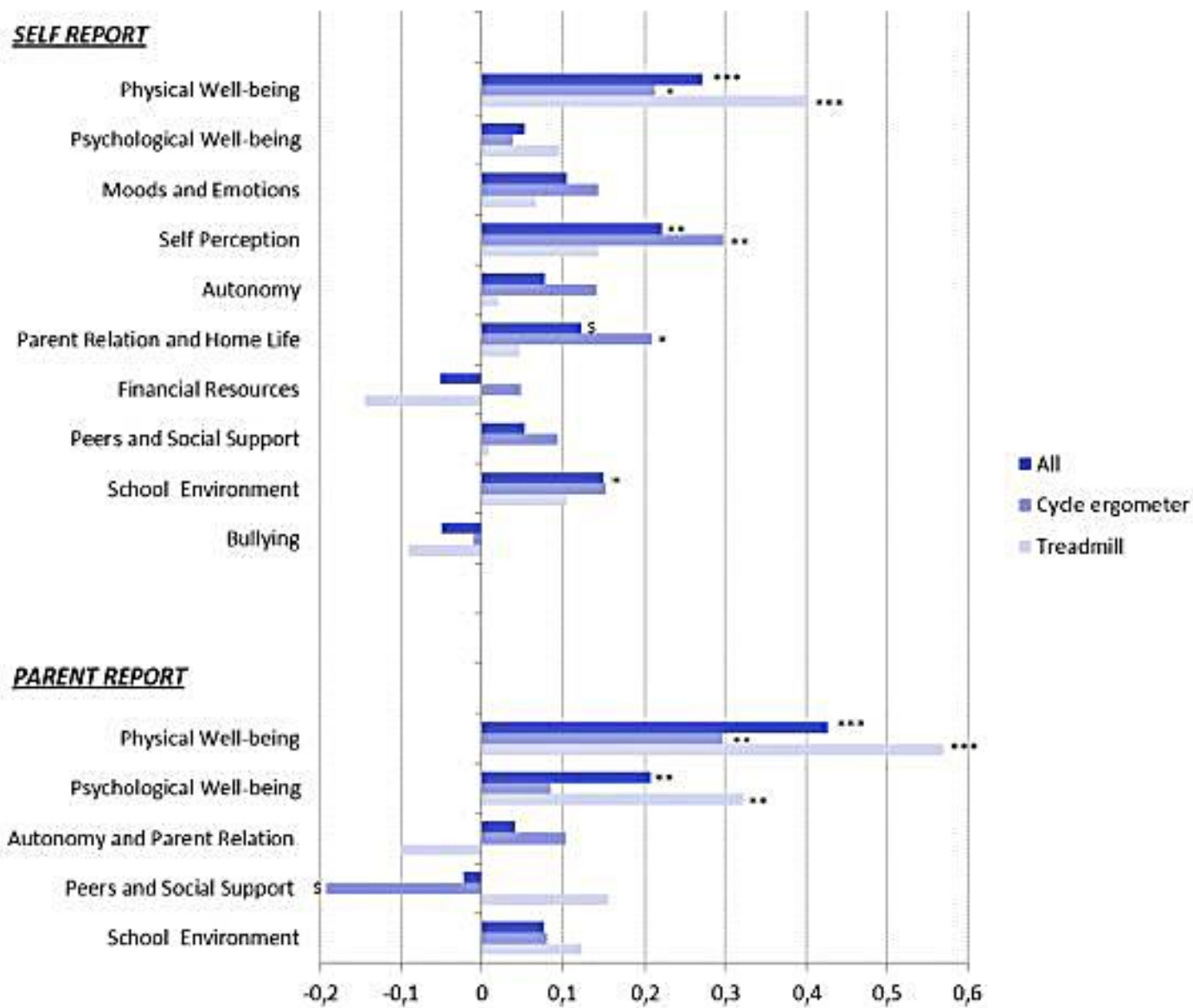

Fig. 1. Correlations between QoL scores (self-reported and parents-reported) and peak VO2. Legend: Significant correlation was denoted by $\$$ for $\mathrm{p} \mathrm{b} 0.10, *$ for $\mathrm{p} \mathrm{b} 0.05$, ** for $\mathrm{p} \mathrm{b} 0.01$ and $* * *$ for $\mathrm{p} \mathrm{b} 0.001$. 
Finally 202 children were included (France $n=106$, Belgium $n=96$ ). Belgian and French populations were not significantly different for all demographic and school characteristics (Table 2). Simple CHD, such as atrial (ASD) and ventricular (VSD) septal defects (15\%), were less represented than complex CHD (Table 3). Sixty-three percent of the patients belonged to severity class 3 or 4 . Only $15 \%$ of children had never had any treatment before inclusion (no drugs, no cardiac surgery, no intervention catheter); $72 \%$ had had at least one cardiac surgery procedure, and $13(7 \%)$ were on drugs only. The two recruiting center populations were comparable for most medical data especially distribution of severity classes, medication and medical devices. However, surgical procedures were significantly more frequent in the Belgian group ( $\mathrm{p}$ b 0.0001).

\subsection{CPET results}

Patients' distribution was homogeneous in terms of age, gender, weight and height between the four severity classes (Table 4) and between CPET procedures (treadmill or cycle ergometer). Few differences were observed between treadmill and cycle ergometer, except for maximum HR, maximum load, and VD/VT ratio (higher values in the treadmill group) (Table 5). Male and female groups were similar in terms of weight, size, and body mass index; male values were higher for peak VO2 and AT, but after normalization with theoretical peak VO2, both variables were not significantly different between males and females (Table 5). A downward trend was observed with increasing CHD severity for peak HR, percentage of predicted $\mathrm{HR}$, peak VO2, percentage of predicted peak VO2, percentage of predicted VO2 at AT, peak oxygen pulse, and conversely for VE/VCO2 slope (Table 4).

\subsection{Correlations between CPET and HR-QoL scores}

The HR-QoL dimensionwhichmost correlated to CPET variableswas "the physical wellbeing", for parents and self-reports and for treadmill and cycle-ergometer procedures. These correlations with the physical well-being scores were particularly significant for three CPET variables: peak $\mathrm{VO} 2$, percentage of predicted peak $\mathrm{VO} 2$ and percentage of predicted $\mathrm{VO} 2$ at AT (presented in Figs. 1, 2 and 3, respectively).

\subsubsection{Self-reported HR-QoL scores (Fig. 4)}

The self-reported physical well-being score correlated with peak VO2 $(r=0.27, \mathrm{p} \mathrm{b} 0.001)$, percentage of predicted peak VO2 $(\mathrm{r}=0.28, \mathrm{p}=0.0001)$, and percentage of predicted VO2 at AT $(r=0.22, \mathrm{p} \mathrm{b} 0.01)$. Such correlations were particularly strong among treadmill CPET for peak VO2 $(r=0.40, \mathrm{p} \mathrm{b} 0.0001)$, percentage of predicted peak VO2 $(\mathrm{r}=0.34, \mathrm{p} \mathrm{b} 0.01)$ and percentage of predicted VO2 at AT $(r=0.26, p=0.01)$.

"School environment" was the "non-physical" dimension that correlated to more CPET variables, with low ( $\mathrm{r}$ b 0.3) correlation rates (percentage of predicted peak VO2, percentage of predicted VO2 at AT, OUES, oxygen pulse at AT, oxygen pulse at peak).

Oxygen pulse at peak was the CPET variable that correlated to more non-physical HR-QoL dimensions, with low ( $\mathrm{r}$ b 0.3 ) correlation rates (financial resources, autonomy, social acceptance). 


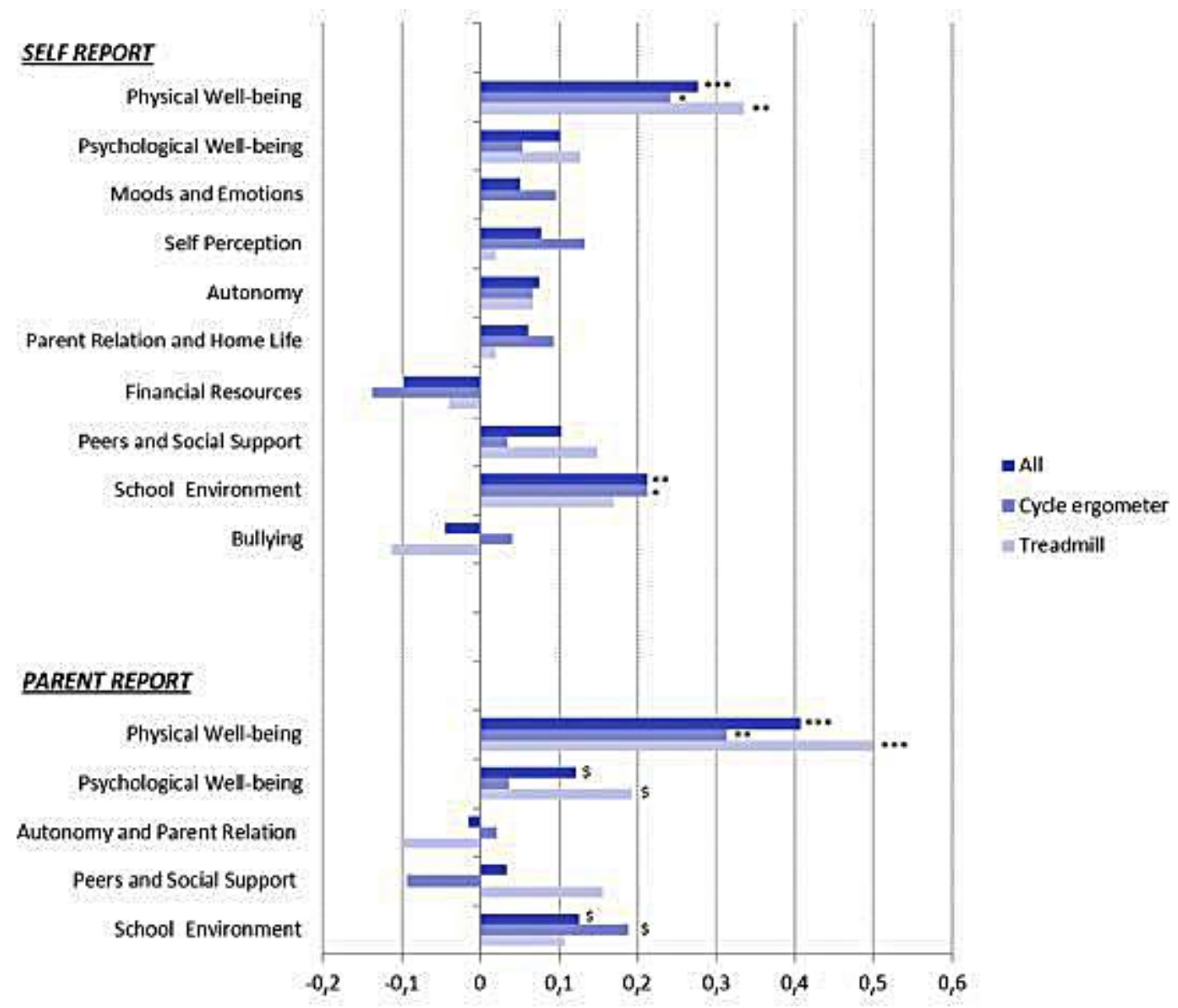

Fig. 2. Correlations between QoL scores (self-reported and parents-reported) and percentage of predicted peak VO2. Legend: Significant correlation was denoted by $\$$ for $p b 0.10, *$ for $p$ $\mathrm{b} 0.05, * *$ for $\mathrm{p} \mathrm{b} 0.01$ and $* * *$ for $\mathrm{p} \mathrm{b} 0.001$.

\subsubsection{Parents-reported HR-QoL scores (Fig. 5)}

The parent-reported physical well-being score correlated to peak VO2 $(\mathrm{r}=0.43, \mathrm{p} \mathrm{b} 0.0001)$, percentage of predicted peak VO2 $(r=0.41, \mathrm{p} \mathrm{b} 0.0001)$ and percentage of predicted $\mathrm{VO} 2$ at $\mathrm{AT}(\mathrm{r}=0.31, \mathrm{p} \mathrm{b} 0.0001)$, and negatively correlated with VD/VT at AT $(\mathrm{r}=-0.29, \mathrm{p} \mathrm{b} 0.001)$ and VD/VT at peak exercise ( $\mathrm{r}=-0.30, \mathrm{p} \mathrm{b}$ 0.0001). Correlations between physical well-being score and VO2 values were significant with both CPET methods, with particularly high correlation coefficients in the treadmill subgroup: peak VO2 ( $\mathrm{r}=0.57, \mathrm{p} \mathrm{b} 0.0001)$, percentage of predicted peak VO2 $(r=0.50, \mathrm{p} \mathrm{b} 0.0001)$ and percentage of predicted VO2 at AT $(\mathrm{r}=0.42$, $\mathrm{p} b$ 0.0001). Moreover, in this treadmill subgroup, negative correlations were observed between parents reported physical well-being scores and VD/VT at AT $(r=-0.32, \mathrm{p} \mathrm{b} 0.01)$ and at peak exercise $(\mathrm{r}=-0.32, \mathrm{p} \mathrm{b} 0.01)$.

Significant but low correlation rates $(\mathrm{r}$ b 0.3$)$ were observed between parents reported psychological well-being, parents reported school environment and peak VO2.

Sensitivity analysis for age and gender showed that the correlations between the physical dimension of the Kidscreen and peak VO2 remained significant and globally concordant with 
the global results excepted for youngest children self-reports (non significant) and for male self-reports ( $\mathrm{r}$ b 0.2) (Table 6).

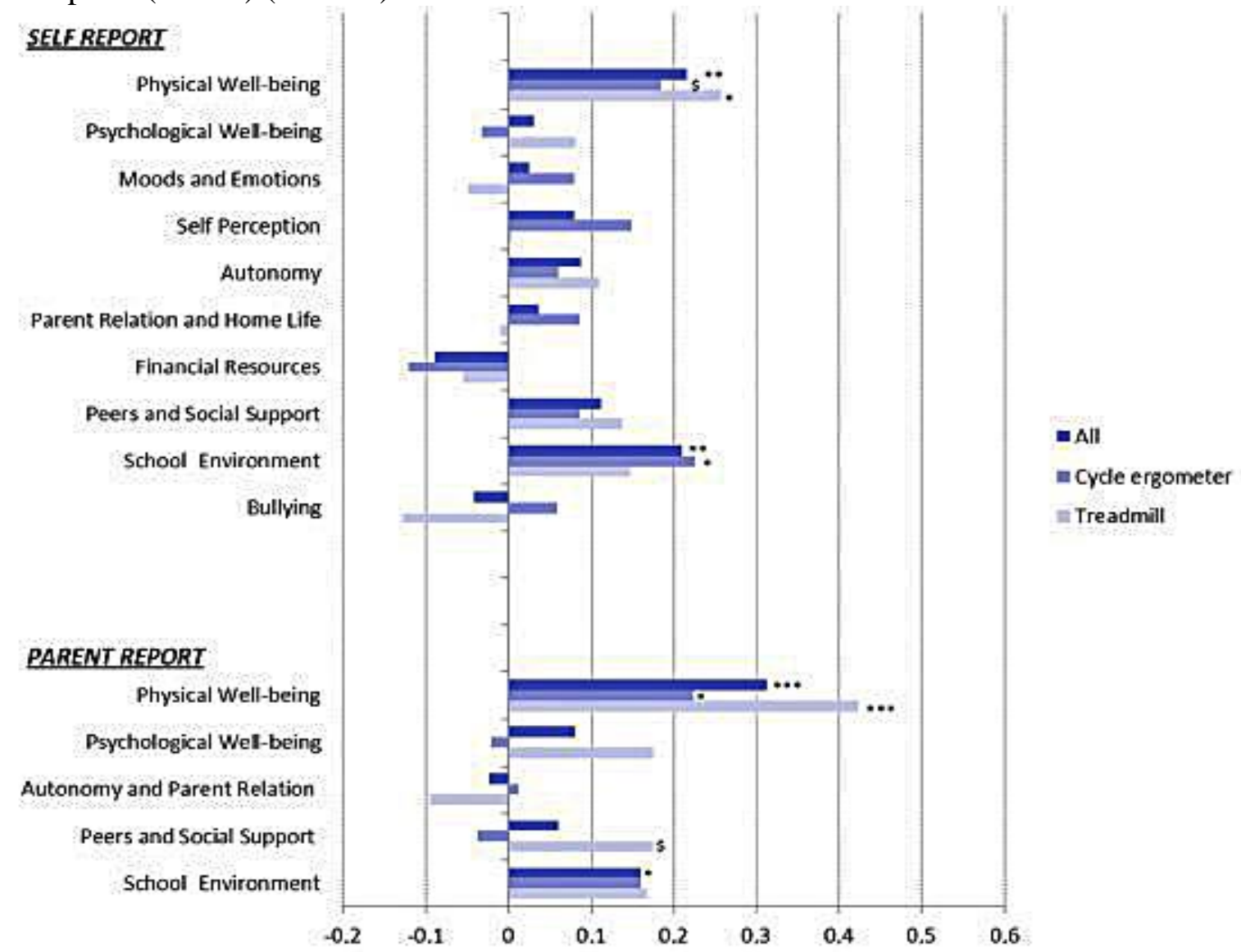

Fig. 3. Correlations between QoL scores (self-reported and parents-reported) and percentage of predicted VO2 at anaerobic threshold. Legend: Significant correlation was denoted by $\$$ for $\mathrm{p} \mathrm{b} 0.10, *$ for $\mathrm{p} \mathrm{b} 0.05, * *$ for $\mathrm{p} \mathrm{b} 0.01$ and $* * *$ for $\mathrm{p} \mathrm{b} 0.001$.

\section{Discussion}

This large pediatric cohort of 202 CHD patients aged 8 to 18 years identified interesting correlations between CPET indices and HR-QoL as reported by both the children themselves and their parents.

The main result lies in the important correlations found between the physical well-being scores of the Kidscreen and the maximum oxygen uptake, with or without normalization. Interestingly, this maximum effort is probably rarely performed in the child's everyday life and is nevertheless related to the HR-QoL which meanwhile mostly reflects daily life. Peak VO2 is considered as a "gold standard" to objectively measure the physical impairment in adult heart failure population [11]. The fact that we found significantly lower peak VO2 values with increasing CHD severity reinforces the idea of such an analogy between pediatric and adult cardiology.

Besides peak VO2, the anaerobic threshold (AT) was well correlated to the physical dimension of the Kidscreen for both self and parents' reports. Moreover we found significantly lower AT values with increasing CHD severity, suggesting the potential 
importance of this variable in pediatric cardiology patients. Low AT in adult population is known to be associated with deconditioning, which may affect many cardiac patients. Therefore, among 4415 CPET performed in adults with CHD, Kempny et al. estimated the AT values to help congenital cardiologists advise patients on activities of daily life, sports participation, and choice of occupation [34]. Besides, adults with CHD and deconditioning are classically eligible to a rehabilitation program aiming to improve physical performances and QoL. However few pediatric centers have such a structured medical care. The correlation found in our study between impaired HR-QoL and low AT among CHD children opens up horizons in pediatric rehabilitation programs. The effects of physical exercise training programs in children and young adults with CHD were recently reviewed: among the 31 published studies only four took the QoL outcome into account [35]. Therefore, when managing transition to adulthood for adolescents with congenital heart diseases [36], the evaluation of the impact of an educational program could use the AT and the HR-QoL before and after rehabilitation as original endpoints. Moreover, it is sometimes difficult to obtain a maximum CPET in the most severe CHD: the AT might then replace the peak VO2 if not reached.

Our study pointed out the correlations between CPET variable and parents-reported HR-QoL scores, which appeared to be stronger than with their children self-reported scores, especially for the physical dimension. Among the youngest children, parents could identify a significant correlation between peak VO2 and the physical dimension of the Kidscreen, while children themselves did not. Moreover, some correlations were also present in other dimensions such as the psychological well-being which correlated with peak VO2 for parents' reports and not for children's. Similarly, some ventilatory parameters such as dead space to tidal volume ratio (VD/VT) correlated to parents reported HR-QoL (physical well-being, school environment) but not to children's reports. Previous QoL studies in pediatric chronic diseases [37-40] and in pediatric cardiology $[17,32,41]$ have shown that proxies underscored HR-QoL compared to the child's own estimation. Our results stated that parents' reported HR-QoL in the physical dimension of the Kidscreen was more related to the actual physical performance of their child as objectively measured by CPET. Moreover, parents seemed to be more accurate in identifying the impact of the CHD on the psychological wellbeing of their child.

This study also considered some other main CPET variables: the VE/ VCO2 slope and the oxygen pulse. Both are classically associated with prognosis in adult heart failure [9-11] and were interestingly also affected by the severity of the CHD in our pediatric cohort. However, we did not find any correlation between the VE/VCO2 slope and any HR-QoL dimension. Similarly, the oxygen pulse did not correlate with the physical well-being but with most of the "non physical" dimensions (school, autonomy, financial resources, social acceptance) for self reports only. One more last controversial CPET variable was finally considered: the oxygen uptake efficiency slope (OUES). Some authors have identified it as a reliable CPET variable when peak VO2 is not reached [26-28]; however in our study, OUES was neither impacted by the severity of the CHD nor related to impaired physical well-being.

When considering the "non-physical" HR-QoL dimensions, we found many correlations between school environment and CPET, for both self and parents' reports. Through these questions related to school, the Kidscreen questionnaire possibly identified some impact of the CHD on these children everyday life. Upon pediatric cardiologists' statements, CHD children might be more brooded by their parents, more stigmatized by their teachers and because of their CHD some of them don't participate to physical activity at school. In most cases, physical activity and sports are authorized under individual pediatric cardiologist's 
prescription $[42,43]$, but these barriers to physical activity for youngsters with CHD put them on the sidelines [44]. Therefore, caregivers, parents and teachers need to consider this potential impact of the CHD on school life.

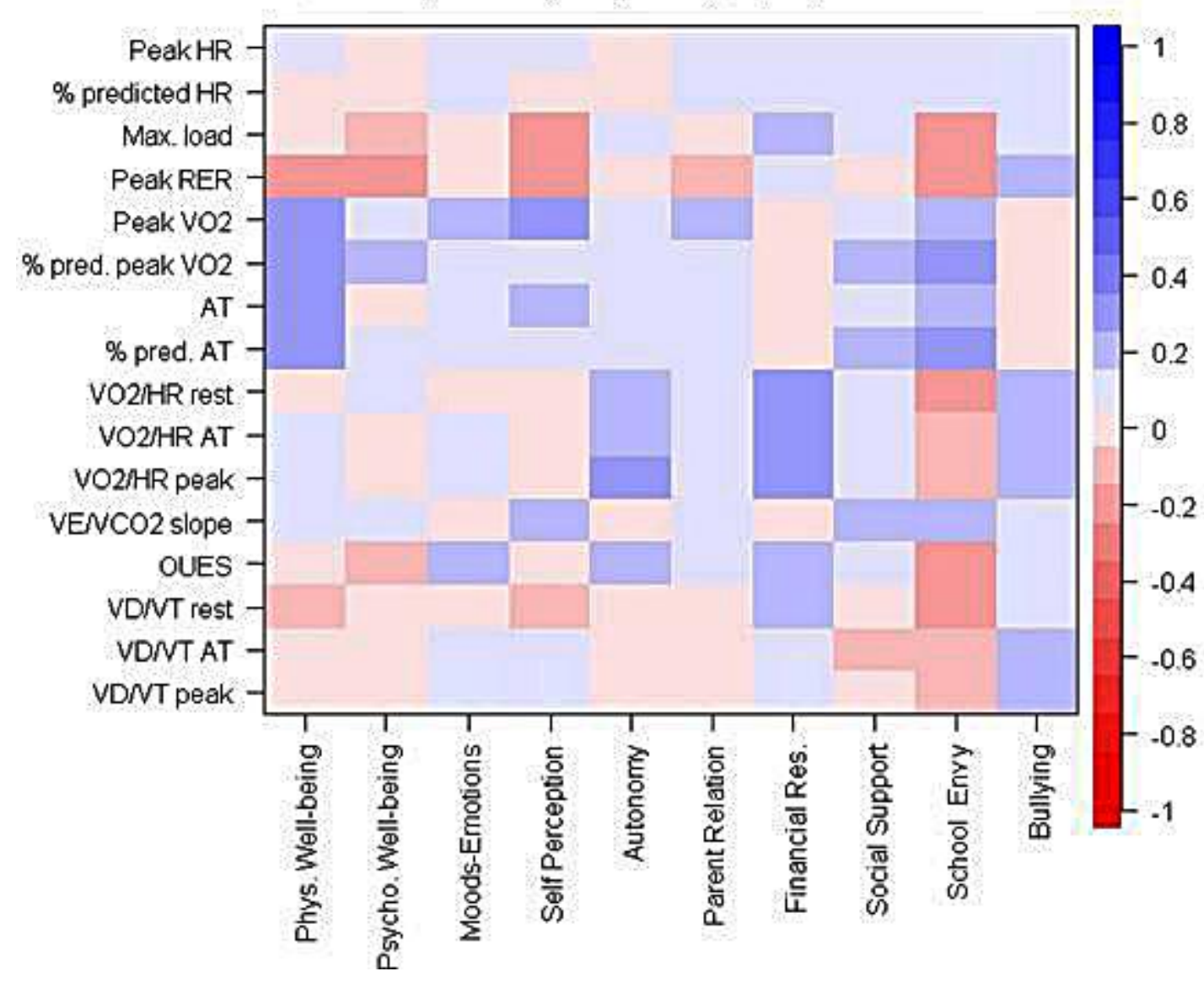

Fig. 4. Correlations between self-reported QoL scores and CEPT. Legend: Correlation matrix between self-reported QoL scores (10 dimensions of the Kidscreen-52) and CPET variables. . (For interpretation of the references to color in this figure legend, the reader is referred to the web version of this article.)

\subsection{Study limitation}

Due to habits of each center on the use of different ergometers, Belgian children performed treadmill CPET and French children performed cycle ergometer CPET. However, the two groups did not differ in terms of age, sex, school characteristics and CHD severity, suggesting limited bias. Moreover, most CPET results were not different between the two methods, especially for the two main variables, i.e. the percentage of predicted oxygen uptake at peak and at anaerobic threshold. Although we cannot overlook a potential center effect, the fact that similar results were found globally and separately in two different populations and Shades of blue indicate increasing positive correlation coefficient; shades of red indicate increasing negative correlation coefficient with two CPET procedures, reinforces the idea of a strong link between the physical well-being HR-QoL dimension and CPET among CHD children. Interestingly, in our cohort, treadmill CPET results were particularly well correlated with HR-QoL dimensions, with a correlation coefficient up to 0.57 between parents-reported 
physical dimension and peak VO2. However, the design of our study does not allow us to conclude that treadmill best reflects the impact of CHD on the child's HR-QoL. Besides, even if the two cultures are very close, the QoL of French and Belgian children cannot be assimilated.

Parents' reports seemed to identify a correlation between CPET and some non-physical HRQoL dimensions such as "psychological wellbeing" and "school environment". We previously showed that these two dimensions were affected by the severity of the CHD in multivariate analysis [17]. Therefore, their association with CPET might only be due to the confounding effect of the severity of the disease. Moreover, it is also possible that children have learned to "cope" with their CHD and don't express any psychological wrong-being [45]. Therefore, even though other dimensions than "the physical well-being" are useful for the follow-up of patients, their use as endpoints in pediatric cardiology clinical trials is subject to many limitations.

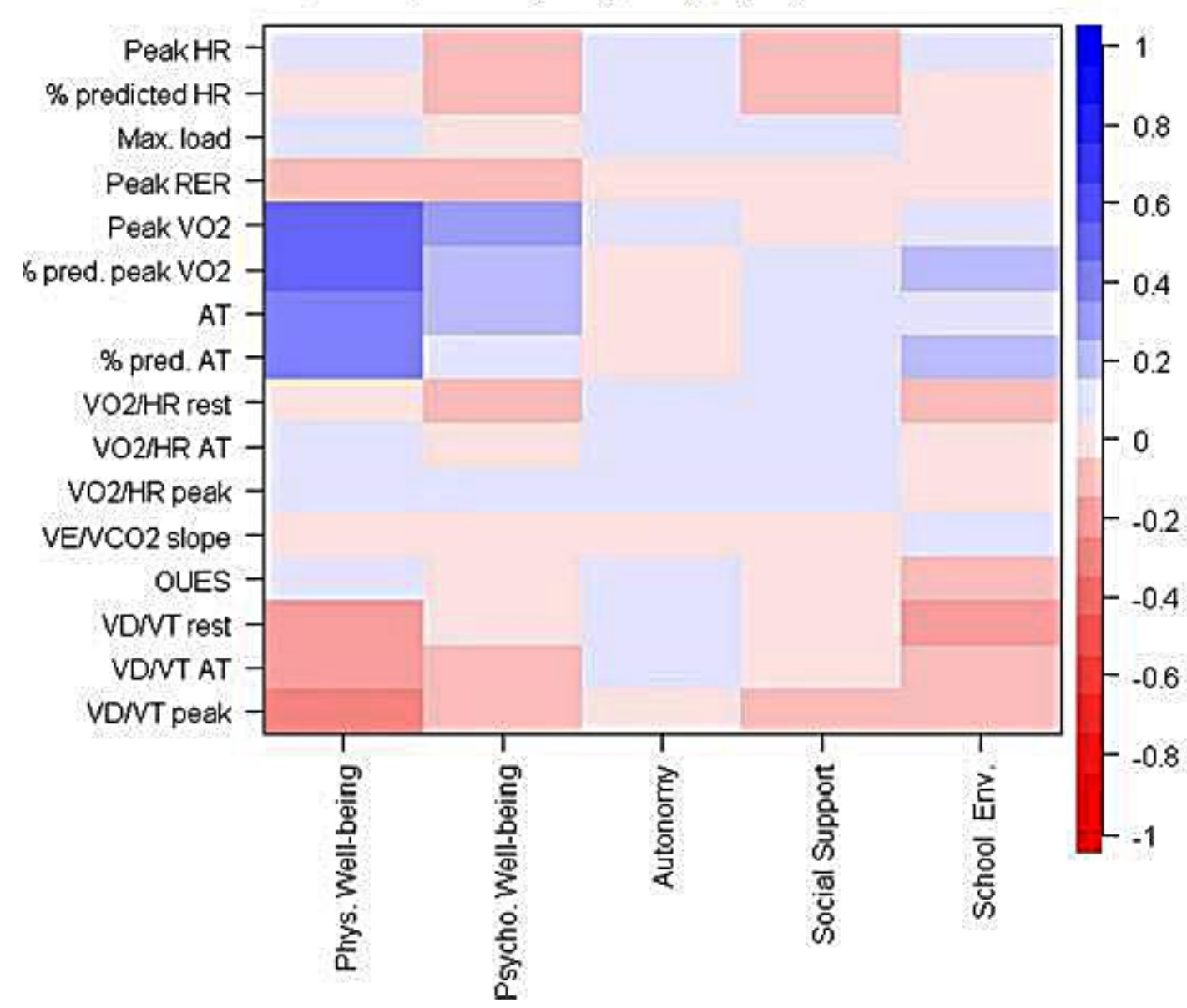

Fig. 5. Correlations between parents-reported QoL scores and CEPT. Legend: Correlation matrix between parents-reported QoL scores (5 dimensions of the Kidscreen-27) and CPET variables. Shades of blue indicate increasing positive correlation coefficient; shades of red indicate increasing negative correlation coefficient. (For interpretation of the references to color in this figure legend, the reader is referred to the web version of this article.) 


\section{Conclusion}

In this large pediatric CHD cohort, peak VO2 and anaerobic threshold were the two CPET variables that best correlated with the physical wellbeing dimension of the Kidscreen HRQoL questionnaire as reported by the children themselves or by their parents. These two CPET variables were significantly lowered with increasing CHD severity. CPET variables appeared to be better correlated with parents reported HR-QoL scores than with children selfreported scores. Precisely, parents' reported HR-QoL in the physical dimension of the Kidscreen was well related to the actual physical performance of their child as objectively measured by CPET. If HR-QoL is involved as a "PRO" in a pediatric cardiology clinical trial, we suggest using parents related physical well-being HR-QoL scores. Further studies are necessary to measure the dynamic aspect of HRQoL in the follow-up of children with CHD.

Table 6 Correlations between peak VO2 and the physical dimension of the Kidscreen.

\begin{tabular}{|c|c|c|c|c|c|c|c|}
\hline & & \multicolumn{3}{|c|}{ Self-reports } & \multicolumn{3}{|c|}{ Parents' reports } \\
\hline & & $r$ & $N$ & $p$ & r & $N$ & $\mathrm{p}$ \\
\hline Overall & & 0.27 & 194 & 0.0001 & 0.43 & 190 & 0.0001 \\
\hline \multirow[t]{4}{*}{ Age groups } & $8-9$ years old & 0.04 & 44 & 0.8 & 0.38 & 45 & 0.01 \\
\hline & $10-12$ years $\mathrm{dd}$ & 033 & 58 & 0.01 & 0.45 & 58 & -0.001 \\
\hline & 13-15 years old & 0.37 & 49 & 0.01 & 0.41 & 47 & 0.005 \\
\hline & $16-18$ years old & 026 & 41 & 0.04 & 0.42 & 39 & 0.007 \\
\hline \multirow[t]{2}{*}{ Gender } & Male & 0.18 & 129 & 0.04 & 0.40 & 126 & -0.0001 \\
\hline & Female & 037 & 65 & 0.003 & 0.39 & 64 & 0.001 \\
\hline
\end{tabular}

Significant p-values are marked in bold.

\section{Funding Montpellier}

University Hospital Clinical Research Program (PHRC) (UF8422) funded this work.

\section{Conflict of interest}

None.

\section{Acknowledgments}

We thank Lorraine Saint-Remy (CPET laboratory technician), Annie Auer (specialist nurse), Anne Cadene (clinical research associate) and Valerie Macioce (medical writer).

\section{References}

[1] F.M. Teixeira, R.M. Coelho, C. Proenca, A.M. Silva, D. Vieira, C. Vaz, et al., Quality of life experienced by adolescents and young adults with congenital heart disease, Pediatr Cardiol 32 (2011) 1132-1138. 
[2] S.D. Anker, S. Agewall, M. Borggrefe, M. Calvert, J. Jaime Caro, M.R. Cowie, et al., The importance of patient-reported outcomes: a call for their comprehensive integration in cardiovascular clinical trials, Eur Heart J 35 (2014) 2001-2009.

[3] P. Moons, W. Budts, S. De Geest, Critique on the conceptualisation of quality of life: a review and evaluation of different conceptual approaches, Int J Nurs Stud 43 (2006) 891-901. [4] T. Bakas, S.M.McLennon, J.S. Carpenter, J.M. Buelow, J.L. Otte, K.M. Hanna, et al., Systematic review of health-related quality of life models, Health Qual Life Outcomes 10 (2012) 134.

[5] M. Bullinger, S. Schmidt, C. Petersen, M. Erhart, U. Ravens-Sieberer, Methodological challenges and potentials of health-related quality of life evaluation in children with chronic health conditions under medical health care, Med Klin 102 (2007) 734-745.

[6] M. Bullinger, U. Ravens-Sieberer, General principles, methods and areas of application of quality of life research in children, Prax Kinderpsychol Kinderpsychiatr 44 (1995) 391-399.

[7] P.H. Wood, Appreciating the consequences of disease: the international classification of impairments, disabilities, and handicaps, WHO Chronicle. 34 (1980) 376-380.

[8] P. Ponikowski, D.P. Francis, M.F. Piepoli, L.C. Davies, T.P. Chua, C.H. Davos, et al., Enhanced ventilatory response to exercise in patients with chronic heart failure and preserved exercise tolerance: marker of abnormal cardiorespiratory reflex control and predictor of poor prognosis, Circulation 103 (2001) 967-972.

[9] R. Poggio, H.C. Arazi, M. Giorgi, S.G. Miriuka, Prediction of severe cardiovascular events by VE/VCO2 slope versus peak VO2 in systolic heart failure: a metaanalysis of the published literature, Am Heart J 160 (2010) 1004-1014.

[10] D.Y. Sue, Excess ventilation during exercise and prognosis in chronic heart failure, Am J Respir Crit Care Med 183 (2011) 1302-1310.

[11] J. Myers, R. Arena, F. Dewey, D. Bensimhon, J. Abella, L. Hsu, et al., A cardiopulmonary exercise testing score for predicting outcomes in patients with heart failure, Am Heart J 156 (2008) 1177-1183.

[12] A. Giardini, A. Hager, C. Pace Napoleone, F.M. Picchio, Natural history of exercise capacity after the Fontan operation: a longitudinal study, Ann Thorac Surg 85 (2008) 818821.

[13] G.P. Diller, A. Giardini, K. Dimopoulos, G. Gargiulo, J.Muller, G. Derrick, et al., Predictors of morbidity and mortality in contemporary Fontan patients: results from a multicenter study including cardiopulmonary exercise testing in 321 patients, Eur Heart J 31 (2010) 3073-3083.

[14] G.P. Diller, K. Dimopoulos, D. Okonko, W. Li, S.V. Babu-Narayan, C.S. Broberg, et al., Exercise intolerance in adult congenital heart disease: comparative severity, correlates, and prognostic implication, Circulation 112 (2005) 828-835.

[15] A. Gratz, J. Hess, A. Hager, Self-estimated physical functioning poorly predicts actual exercise capacity in adolescents and adultswith congenital heart disease, Eur Heart J 30 (2009) 497-504.

[16] A. Hager, J. Hess, Comparison of health related quality of life with cardiopulmonary exercise testing in adolescents and adults with congenital heart disease, Heart 91 (2005) 517 520.

[17] P. Amedro, R. Dorka, S. Moniotte, S. Guillaumont, A. Fraisse, B. Kreitmann, et al., Quality of life of children with congenital heart diseases: a multicenter controlled crosssectional study, Pediatr Cardiol (2015).

[18] U. Ravens-Sieberer, A. Gosch, L. Rajmil, M. Erhart, J. Bruil,W. Duer, et al., Kidscreen52 quality-of-life measure for children and adolescents, Exp. Rev. Pharmacoeconomics Outcomes Res. 5 (2005) 353-364. 
[19] U. Ravens-Sieberer, A. Gosch, L. Rajmil, M. Erhart, J. Bruil, M. Power, et al., The KIDSCREEN-52 quality of life measure for children and adolescents: psychometric results from a cross-cultural survey in 13 European countries, Value Health 11 (2008) 645-658. [20] U. Ravens-Sieberer, M. Herdman, J. Devine, C. Otto, M. Bullinger, M. Rose, et al., The European KIDSCREEN approach to measure quality of life and well-being in children: development, current application, and future advances, Qual. Life Res. Int. J. Qual. Life Asp. Treat. Care Rehab. 23 (2014) 791-803.

[21] U. Ravens-Sieberer, P. Auquier, M. Erhart, A. Gosch, L. Rajmil, J. Bruil, et al., The KIDSCREEN-27 quality of life measure for children and adolescents: psychometric results from a cross-cultural survey in 13 European countries, Qual. Life Res. Int. J. Qual. Life Asp. Treat. Care Rehab. 16 (2007) 1347-1356.

[22] S. Robitail, U. Ravens-Sieberer, M.C. Simeoni, L. Rajmil, J. Bruil,M. Power, et al., Testing the structural and cross-cultural validity of the KIDSCREEN-27 quality of life questionnaire, Qual. Life Res. Int. J. Qual. Life Asp. Treat. Care Rehab. 16 (2007) 13351345.

[23] A. Zapletal, E.K. Motoyama, K.P. Van DeWoestijne, V.R. Hunt, A. Bouhuys,Maximum expiratory flow-volume curves and airway conductance in children and adolescents, J Appl Physiol 26 (1969) 308-316.

[24] T. Takken, A.C. Blank, E.H. Hulzebos, M. van Brussel,W.G. Groen, P.J. Helders, Cardiopulmonary exercise testing in congenital heart disease: equipment and test protocols, Neth Hear J 17 (2009) 339-344.

[25] W.L. Beaver, K. Wasserman, B.J. Whipp, A new method for detecting anaerobic threshold by gas exchange, J Appl Physiol 60 (1986) 2020-2027.

[26] R. Baba, M. Nagashima, M. Goto, Y. Nagano, M. Yokota, N. Tauchi, et al., Oxygen uptake efficiency slope: a new index of cardiorespiratory functional reserve derived from the relation between oxygen uptake and minute ventilation during incremental exercise, J Am Coll Cardiol 28 (1996) 1567-1572.

[27] B.C. Bongers, H.J. Hulzebos, A.C. Blank, M. van Brussel, T. Takken, The oxygen uptake efficiency slope in children with congenital heart disease: construct and group validity, Eur J Cardiovasc Prev Rehabil 18 (2011) 384-392.

[28] A. Giardini, S. Specchia, G. Gargiulo, D. Sangiorgi, F.M. Picchio, Accuracy of oxygen uptake efficiency slope in adults with congenital heart disease, Int J Cardiol 133 (2009) 7479.

[29] D.M. Cooper, C. Berry, N. Lamarra, K. Wasserman, Kinetics of oxygen uptake and heart rate at onset of exercise in children, J Appl Physiol 59 (1985) 211-217.

[30] D.M. Cooper, D. Weiler-Ravell, B.J. Whipp, K. Wasserman, Growth-related changes in oxygen uptake and heart rate during progressive exercise in children, Pediatr Res 18 (1984) 845-851.

[31] D.M. Cooper, D.Weiler-Ravell, B.J.Whipp, K.Wasserman, Aerobic parameters of exercise as a function of body size during growth in children, J Appl Physiol Respir Environ Exerc Physiol 56 (1984) 628-634.

[32] K. Uzark, K. Jones, J. Slusher, C.A. Limbers, T.M. Burwinkle, J.W. Varni, Quality of life in children with heart disease as perceived by children and parents, Pediatrics 121 (2008) e1060-e1067.

[33] L. Houyel, B. Khoshnood, R.H. Anderson, N. Lelong, A.C. Thieulin, F. Goffinet, et al., Population-based evaluation of a suggested anatomic and clinical classification of congenital heart defects based on the International Paediatric and Congenital Cardiac Code, Orphanet $\mathbf{J}$ Rare Dis 6 (2011) 64.

[34] A. Kempny, K. Dimopoulos, A. Uebing, P. Moceri, L. Swan, M.A. Gatzoulis, et al., Reference values for exercise limitations among adults with congenital heart disease. Relation 
to activities of daily life-single centre experience and review of published data, Eur Heart J 33 (2012) 1386-1396.

[35] N. Duppen, T. Takken,M.T. Hopman, A.D. ten Harkel, K. Dulfer, E.M. Utens, et al., Systematic review of the effects of physical exercise training programmes in children and young adultswith congenital heart disease, Int J Cardiol 168 (2013) 1779-1787.

[36] C. Sable, E. Foster, K. Uzark, K. Bjornsen, M.M. Canobbio, H.M. Connolly, et al., Best practices in managing transition to adulthood for adolescents with congenital heart disease: the transition process andmedical and psychosocial issues: a scientific statement from the American Heart Association, Circulation 123 (2011) 1454-1485.

[37] C. Eiser, R. Morse, Can parents rate their child's health-related quality of life? Results of a systematic review, Qual. Life Res. Int. J. Qual. Life Asp. Treat. Care Rehab. 10 (2001) 347 357.

[38] J. Cremeens, C. Eiser, M. Blades, Factors influencing agreement between child selfreport and parent proxy-reports on the Pediatric Quality of Life Inventory 4.0 (PedsQL) generic core scales, Health Qual Life Outcomes 4 (2006) 58.

[39] V. Matziou, P. Perdikaris, D. Feloni,M.Moschovi, K. Tsoumakas, A. Merkouris, Cancer in childhood: children's and parents' aspects for quality of life, Eur J Oncol Nurs 12 (2008) 209-216.

[40] P. Upton, J. Lawford, C. Eiser, Parent-child agreement across child health-related quality of life instruments: a review of the literature, Qual Life Res Int J Qual Life Asp Treat Care Rehab 17 (2008) 895-913.

[41] B.S. Marino, R.S. Tomlinson, D. Drotar, E.S. Claybon, A. Aguirre, R. Ittenbach, et al., Quality-of-life concerns differ among patients, parents, and medical providers in children and adolescents with congenital and acquired heart disease, Pediatrics 123 (2009) e708-e715.

[42] B.J. Maron, B.R. Chaitman, M.J. Ackerman, A. Bayes de Luna, D. Corrado, J.E.

Crosson, et al., Recommendations for physical activity and recreational sports participation for young patients with genetic cardiovascular diseases, Circulation 109 (2004) 2807-2816. [43] J.H. Mitchell, B.J. Maron, S.E. Epstein, 16th Bethesda Conference: cardiovascular abnormalities in the athlete: recommendations regarding eligibility for competition. October 3-5, 1984, J Am Coll Cardiol 6 (1985) 1186-1232.

[44] F. Moola, C. Fusco, J.A. Kirsh, The perceptions of caregivers toward physical activity and health in youth with congenital heart disease, Qual Health Res 21 (2011) 278-291.

[45] B. Latal, S. Helfricht, J.E. Fischer, U. Bauersfeld, M.A. Landolt, Psychological adjustment and quality of life in children and adolescents following open-heart surgery for congenital heart disease: a systematic review, BMC Pediatr 9 (2009) 6. 